\title{
EXTREMAL FAMILIES OF CUBIC THUE EQUATIONS
}

\author{
MICHAEL A. BENNETT AND AMIR GHADERMARZI \\ On the occasion of Axel Thue's 150th birthday
}

\begin{abstract}
We exactly determine the integral solutions to a previously untreated infinite family of cubic Thue equations of the form $F(x, y)=1$ with at least 5 such solutions. Our approach combines elementary arguments, with lower bounds for linear forms in logarithms and lattice-basis reduction.
\end{abstract}

\section{INTRODUCTION}

If $F(x, y)$ is an irreducible binary form (i.e. homogenous polynomial) with integer coefficients and degree $n \geq 3$, and $m$ is a nonzero integer, the Diophantine equation $F(x, y)=m$ is called a Thue equation in honour of Axel Thue 38 who proved, more than a century ago, that the associated number of solutions in integers $x$ and $y$ is finite. Such equations arise somewhat naturally in a wide variety of number theoretic contexts, including questions about the existence of primitive divisors in binary recurrence sequences and in the only known algorithm (in the strict sense of the term) for finding integral points on genus one curves over $\mathbb{Q}$. Our current understanding of such equations is a rather refined one and we now have excellent upper bounds upon their number of solutions, depending only on $n$ and $m$ (and, in particular, not upon the coefficients of the form; see Evertse [15] and Bombieri and Schmidt [11). These bounds arise from treating the apparently special case $m=1$ and applying "lifting" arguments, dating back to Lagrange (see page 673 of Dickson [14] and also Mahler [23]).

The case when $n=3$, i.e. that of cubic forms, is a relatively simple one and is well understood. This is primarily because cubic Thue equations may be attacked with a wide variety of approaches that do not apparently generalize to those of higher degree. The particular case of cubic Thue equations of the shape $F(x, y)=1$ where the form $F$ has a negative discriminant is especially simple, as the presence of a single fundamental unit in the corresponding cubic field makes application of Skolem's $p$-adic method or similar local techniques relatively routine. Using such an approach (where we write $N_{F}$ for the number of integral solutions to the equation $F(x, y)=1$ and $D_{F}$ for the discriminant of $F$ ), Delone [13] and Nagell 30] independently proved

Theorem 1.1. (Delone and Nagell) If $F$ is an irreducible binary cubic form with integer coefficients and $D_{F}<0$, then $N_{F} \leq 5$. Moreover, if $N_{F}=5$, then $F$ is $G L_{2}(\mathbb{Z})$-equivalent to

$$
x^{3}-x y^{2}+y^{3},
$$

1991 Mathematics Subject Classification. Primary 11D25, Secondary 11E76.

Key words and phrases. Thue equations, binary cubic forms.

The first author was supported in part by NSERC. 
with $D_{F}=-23$ and, if $N_{F}=4$, then $F$ is $G L_{2}(\mathbb{Z})$-equivalent to either

$$
x^{3}+x y^{2}+y^{3} \text { or } x^{3}-x^{2} y+x y^{2}+y^{3},
$$

with discriminant -31 or -44 , respectively.

In the case of cubic forms of positive discriminant, the situation is rather more complicated and there are a number of forms for which $N_{F}$ exceeds 5 . The following table collects representatives of all known equivalence classes of irreducible cubic forms for which $N_{F} \geq 6$.

\begin{tabular}{|cccl|}
\hline$F(x, y)$ & $D_{F}$ & $N_{F}$ & References \\
\hline$x^{3}-x^{2} y-2 x y^{2}+y^{3}$ & 49 & 9 & {$[6],[16],[22,[35]$} \\
$x^{3}-3 x y^{2}+y^{3}$ & 81 & 6 & {$[16],[22],[39]$} \\
$x^{3}-4 x y^{2}+y^{3}$ & 229 & 6 & {$[12],[16],[35]$} \\
$x^{3}-5 x y^{2}+3 y^{3}$ & 257 & 6 & {$[16]$} \\
$x^{3}+2 x^{2} y-5 x y^{2}+y^{3}$ & 361 & 6 & {$[16]$} \\
\hline
\end{tabular}

Presumably, we always have $N_{F} \leq 9$. A striking theorem of Okazaki 32 is that $N_{F} \leq 7$ provided $D_{F}$ is suitable large (see also Akhtari [1]); we have $N_{F} \leq 10$ in all cases, via an old result of the first author [10].

A stronger conjecture, due originally to Nagell 31] and subsequently refined by Pethö [33] and Lippok [21, is that the forms in the above table are, up to equivalence, the only irreducible cubics with $N_{F} \geq 6$, so that, for all other classes, we have $N_{F} \leq 5$. If true, this upper bound for $N_{F}$ is sharp, as we know of a number of infinite families of cubic forms where the number of integer solutions to the corresponding equation $F(x, y)=1$ is at least 5 . Let us define

$$
\begin{gathered}
F_{1, t}(x, y)=x^{3}-(t+1) x^{2} y+t x y^{2}+y^{3}, \\
F_{2, t}(x, y)=x^{3}-t^{2} x y^{2}+y^{3}
\end{gathered}
$$

and

$$
F_{3, t}(x, y)=x^{3}-\left(t^{4}-t\right) x^{2} y+\left(t^{5}-2 t^{2}\right) x y^{2}+y^{3} .
$$

In each case, $F_{i}(x, y)$ is irreducible over $\mathbb{Q}[x, y]$, with $N_{F_{i, t}} \geq 5$, at least provided we exclude "small" values of the integer parameter $t$ (the assumption that $|t|>2$ is sufficient).

It is plausible to believe that (1.1), (1.2) and (1.3) represent the only infinite cubic families for which the corresponding Thue equation $F(x, y)=1$ has five or more integral solutions, partially explaining our (misleading) title. The evidence does not admittedly seem especially compelling, one way or the other. That being said, we will take this opportunity to solve those equations corresponding to the third family, which has not previously been treated in the literature. We note that the equation $F_{1, t}(x, y)=1$ was completely solved by Lee [20, Mignotte and Tzanakis [29] and Mignotte [27, while $F_{2, t}(x, y)=1$ has been treated for $|t| \geq 1.35 \cdot 10^{14}$ by Wakabayashi 41]. We prove

Theorem 1.2. If $t$ is an integer, then the Diophantine equation

$$
F_{3, t}(x, y)=x^{3}-\left(t^{4}-t\right) x^{2} y+\left(t^{5}-2 t^{2}\right) x y^{2}+y^{3}=1,
$$

has only the integer solutions

$$
(x, y) \in\left\{(1,0),(0,1),(t, 1),\left(t^{4}-2 t, 1\right),\left(1-t^{3}, t^{8}-3 t^{5}+3 t^{2}\right)\right\},
$$


unless $t=-1$, in which case there is an additional solution given by $(x, y)=(6,-5)$.

Our argument follows the now-traditional approach originated by Thomas [36], 37. A nice survey of families of Thue equations solved to date (by these and other methods) can be found in Heuberger [17] (see also [18] and [19] for other good expositions along these lines).

\section{The Equation $F_{3, t}(x, y)=1$ : Units in CUBIC Fields}

For the remainder of the paper, we will concern ourselves with the parametric equation (1.4). Note that $N_{F_{3, t}} \geq 5$ (with solutions given in the statement of Theorem 1.2) for $t \notin\{0,1\}$. We have

$$
D_{F_{3, t}}=t^{18}-10 t^{15}+41 t^{12}-90 t^{9}+102 t^{6}-40 t^{3}-27,
$$

which is positive, except for $t \in\{0,1\}$. That $N_{F_{3, t}} \geq 5$ was noted by Ziegler [42] who observed that one also has $N_{F_{4, t}} \geq 5$, for the families of forms given by

$$
F_{4, t}(x, y)=x^{3}-\left(t^{4}+4 t\right) x^{2} y+\left(t^{5}+3 t^{2}\right) x y^{2}+y^{3} .
$$

We have $F_{3,-t}(x, y) \sim F_{4, t}(x, y)$ under $G L_{2}(\mathbb{Z})$ action, since $F_{3,-t}(x-t y, y)=$ $F_{4, t}(x, y)$.

Let us suppose for the next few sections that $t \geq 10$; our argument for negative values of $t$ is very similar and will not be reproduced here, while the treatment of "small" values of $t$ requires rather different techniques. Defining $P(x)=F_{3, t}(x, 1)$, then $P(x)$ has three real roots, which we denote by $\theta_{1}<\theta_{2}<\theta_{3}$. By studying the sign of $P(x)$, we can deduce the following expressions for these roots :

(2.1) $\theta_{1}=-\frac{1}{t^{5}}-\frac{2}{t^{8}}-\frac{\kappa_{1}}{t^{11}}, \theta_{2}=t+\frac{1}{t^{5}}+\frac{3}{t^{8}}+\frac{\kappa_{2}}{t^{11}}$ and $\theta_{3}=t^{4}-2 t-\frac{1}{t^{8}}-\frac{\kappa_{3}}{t^{11}}$.

Here, the $\kappa_{i}$ are certain real numbers with $\kappa_{1} \in(3,3.1), \kappa_{2} \in(8,8.03)$ and $\kappa_{3} \in$ $(5,5.02)$.

Suppose that $(x, y)$ is a solution to equation (1.4). It follows, for each $i \in\{1,2,3\}$, that $x-y \theta_{i}$ is a unit in $\mathbb{Q}\left(\theta_{i}\right)$ and hence, since Lemma 4.11 of 37 implies that $t-\theta_{i}$ and $\theta_{i}$ form a pair of fundamental units in $\mathbb{Q}\left(\theta_{i}\right)$, we may write

$$
x-y \theta_{i}=(-1)^{\delta}\left(t-\theta_{i}\right)^{n} \theta_{i}^{-m} \text { for } m, n \in \mathbb{Z}, \delta \in\{0,1\} .
$$

In particular, we have

$$
\frac{x-y \theta_{3}}{x-y \theta_{2}}=\left(\frac{t-\theta_{3}}{t-\theta_{2}}\right)^{n}\left(\frac{\theta_{3}}{\theta_{2}}\right)^{-m}>0,
$$

and so, from the fact that

$$
\left(x-y \theta_{1}\right)\left(x-y \theta_{2}\right)\left(x-y \theta_{3}\right)=1,
$$

we conclude that $x-y \theta_{1}>0$ (whereby at least one of $x$ and $y$ is positive).

If $|y| \leq 1$, we observe after a little work, since we assume $t \geq 10$, that

$$
(x, y) \in\left\{(1,0),(0,1),(t, 1),\left(t^{4}-2 t, 1\right)\right\} .
$$

Let us therefore suppose that $(x, y)$ is a solution in integers to equation (1.4), with $|y| \geq 2$. A routine calculation ensures, provided $t \geq 10$, that necessarily $x / y$ lies in one of the intervals

$$
I_{1}=\left(-\frac{1.13}{t^{5}}, \frac{-1+\frac{1}{|y|^{3}}}{t^{5}}\right), I_{2}=\left(t+\frac{1-\frac{1}{|y|^{3}}}{t^{5}}, t+\frac{1.13}{t^{5}}\right)
$$


or

$$
I_{3}=\left(t^{4}-2 t-\frac{1.13}{t^{8}}, t^{4}-2 t-\frac{1-\frac{1}{|y|^{3}}}{t^{8}}\right) .
$$

As is readily observed, these intervals are disjoint and hence we will call solutions $(x, y)$ to equation (1.4) with $x / y$ in $I_{1}, I_{2}$ and $I_{3}$ solutions of types I, II and III, respectively. It is easy to show (and valuable to note, for later use) that $\left|x-y \theta_{i}\right|<1$ for $i=1,2$ or 3 , provided $(x, y)$ is a solution of type I, II or III, respectively.

From here, we will proceed as follows. We first will use elementary arguments and a careful analysis of equation (2.3) to deduce lower bounds upon $\max \{|m|,|n|\}$ in (2.2). We then combine this information with lower bounds upon

$$
\left|\frac{x-y \theta_{i}}{x-y \theta_{j}}\right|
$$

for suitably chosen pairs $(i, j)$, depending on the solution type of $(x, y)$. The latter bounds arise from invoking lower bounds for linear forms in logarithms of algebraic numbers à la Baker and yield upper bounds for $t$. Finally, we appeal to a classical lemma of Baker and Davenport [5] (in essence, a simple version of Lenstra-LenstraLovacz lattice basis reduction) to treat (most of) the remaining values of $t$.

\section{UPPER BOUNDS FOR $\max \{|m|,|n|\}$ IN EQUATION (2.3)}

In general, it is always possible to reduce the problem of solving families of Thue equations to that of treating unit equations similar to (2.2). A crucial step in solving such families is to ensure suitably rapid growth (Thomas 37] terms this stable growth) of the exponents of the fundamental units. That is the content of this section. Our arguments vary somewhat depending on the solution type of $(x, y)$; we will treat each in turn.

3.1. Solutions of type I. Let us suppose first that $x / y \in I_{1}$, i.e. that $x / y$ satisfies

$$
-\frac{1.13}{t^{5}}<\frac{x}{y}<\frac{-1+\frac{1}{|y|^{3}}}{t^{5}}
$$

If we have $y<0$ and $x>0$ then from $x-y \theta_{1}>0$, it follows that $x / y \in\left(-\frac{1.13}{t^{5}}, \theta_{1}\right)$. From (2.1), we thus have

$$
\frac{t^{3}-2+\frac{1}{t^{6}}+\frac{2-\kappa_{3}}{t^{9}}+\frac{\kappa_{1}}{t^{12}}}{1+\frac{2.13}{t^{6}}+\frac{\kappa_{2}}{t^{9}}}<\frac{x-y \theta_{3}}{x-y \theta_{2}}<\frac{t^{3}-2+\frac{1.13}{t^{6}}-\frac{\kappa_{3}}{t^{9}}}{1+\frac{2}{t^{6}}+\frac{2+\kappa_{2}}{t^{9}}+\frac{\kappa_{1}}{t^{12}}} .
$$

If, on the other hand, we have $y>0$ (so that $y \geq 2$ ) and $x<0$, then $x / y \in$ $\left(\theta_{1}, \frac{-0.875}{t^{5}}\right)$, whereby

$$
\frac{t^{3}-2+\frac{0.875}{t^{6}}-\frac{\kappa_{3}}{t^{9}}}{1+\frac{2}{t^{6}}+\frac{2+\kappa_{2}}{t^{9}}+\frac{\kappa_{1}}{t^{12}}}<\frac{x-y \theta_{3}}{x-y \theta_{2}}<\frac{t^{3}-2+\frac{1}{t^{6}}+\frac{2-\kappa_{3}}{t^{9}}+\frac{\kappa_{1}}{t^{12}}}{1+\frac{1.875}{t^{6}}+\frac{\kappa_{2}}{t^{9}}} .
$$

In either case, we thus may write

$$
\frac{x-y \theta_{3}}{x-y \theta_{2}}=t^{3}-2-\frac{\kappa_{4}}{t^{3}},
$$

where $\kappa_{4} \in(1.8,2.2)$. Arguing similarly, we find that

$$
\log \left(\frac{t-\theta_{3}}{t-\theta_{2}}\right)=9 \log t-\frac{6}{t^{3}}-\frac{\kappa_{5}}{t^{6}}
$$


and

$$
\log \left(\theta_{3} / \theta_{2}\right)=3 \log t-\frac{2}{t^{3}}-\frac{\kappa_{6}}{t^{6}}
$$

$$
\log \left(\frac{x-y \theta_{3}}{x-y \theta_{2}}\right)=3 \log t-\frac{2}{t^{3}}-\frac{\kappa_{7}}{t^{6}},
$$

where $\kappa_{5} \in(7.99,8.03), \kappa_{6} \in(3,3.01)$ and $\kappa_{7} \in(3.8,4.3)$.

We may thus conclude from (2.3) that

$$
k:=3 n-m-1=\frac{1}{3 \log t}\left(\frac{6 n-2 m-2}{t^{3}}+\frac{\kappa_{5} n-\kappa_{6} m-\kappa_{7}}{t^{6}}\right) .
$$

If $k=0$ (so that $m=3 n-1$ ), then it follows that

$$
0=\kappa_{5} n-\kappa_{6}(3 n-1)-\kappa_{7}=\left(\kappa_{5}-3 \kappa_{6}\right) n+\kappa_{6}-\kappa_{7}
$$

and so

$$
n=\frac{\kappa_{6}-\kappa_{7}}{3 \kappa_{6}-\kappa_{5}} \in(-1.25,-0.8), \text { i.e. } n=-1, m=-4 .
$$

Equation (2.2) thus implies, after a little work, that

$$
(x, y)=\left(1-t^{3}, t^{8}-3 t^{5}+3 t^{2}\right) .
$$

We may thus suppose that $k$ is a nonzero integer whence, from equation (3.2), we have

$$
k\left(3 t^{6} \log t-2 t^{3}\right)=\kappa_{5} n-\kappa_{6} m-\kappa_{7}=\frac{\kappa_{5}}{3} k+\frac{\kappa_{5}-3 \kappa_{6}}{3} m+\frac{\kappa_{5}-3 \kappa_{7}}{3} .
$$

It follows that

$$
m=\frac{k}{\kappa_{5}-3 \kappa_{6}}\left(9 t^{6} \log t-6 t^{3}-\kappa_{5}\right)+\frac{3 \kappa_{7}-\kappa_{5}}{\kappa_{5}-3 \kappa_{6}} .
$$

We may conclude from this that $m$ and $k$ are necessarily of opposite sign and, since $t \geq 10$, that

$$
|m|>8.6|k| t^{6} \log t
$$

From $k=3 n-m-1, m$ and $n$ thus have the same sign whereby, considering (2.2) and the fact that $\left|x-y \theta_{1}\right|<1$, we may conclude that $m<n<0,|m|>3|n|$ and, since $k$ is nonzero,

$$
|m|=\max \{|m|,|n|\}>8.6 t^{6} \log t .
$$

3.2. Solutions of type II. Let us next suppose that $x / y$ satisfies

$$
t+\frac{1}{t^{6}}<\frac{x}{y}<t+\frac{2}{t^{5}}
$$

Since at least one of $x$ and $y$ is positive, it follows that both are positive. We therefore have (again appealing to (2.1) and (2.2))

$$
x-y \theta_{1}>0, \quad x-y \theta_{2}<0 \text { and } x-y \theta_{3}<0 .
$$

It follows that

$$
0>\frac{x-y \theta_{3}}{x-y \theta_{1}}=\left(\frac{t-\theta_{3}}{t-\theta_{1}}\right)^{n}\left(\frac{\theta_{3}}{\theta_{1}}\right)^{-m}
$$

and hence $n$ and $m$ are of opposite parity.

Using the inequalities in (2.1) and (3.5), we find that

$$
\frac{t^{3}-3-\frac{2}{t^{6}}-\frac{\kappa_{3}}{t^{9}}}{1+\frac{3}{t^{6}}+\frac{2}{t^{9}}+\frac{\kappa_{1}}{t^{12}}}<\frac{y \theta_{3}-x}{x-y \theta_{1}}<\frac{t^{3}-3-\frac{1}{t^{7}}-\frac{\kappa_{3}}{t^{9}}}{1+\frac{1}{t^{6}}+\frac{1}{t^{7}}+\frac{2}{t^{9}}+\frac{\kappa_{1}}{t^{12}}}
$$


and hence

Similarly,

$$
\frac{y \theta_{3}-x}{x-y \theta_{1}}=t^{3}-3-\frac{\kappa_{8}}{t^{3}}, \quad \text { where } \kappa_{8} \in(0,3.1)
$$

$$
\frac{\theta_{3}-t}{t-\theta_{1}}=t^{3}-3-\frac{\kappa_{9}}{t^{3}}, \text { where } \kappa_{9} \in(0,1.1)
$$

and

We thus have

$$
\frac{\theta_{3}}{\left|\theta_{1}\right|}=t^{9}-4 t^{6}+\kappa_{10} t^{3} \text { with } \kappa_{10} \in(4.9,5) \text {. }
$$

$$
\log \left(t^{3}-3-\frac{\kappa_{8}}{t^{3}}\right)=n \log \left(t^{3}-3-\frac{\kappa_{9}}{t^{3}}\right)-m \log \left(t^{9}-4 t^{6}+\kappa_{10} t^{3}\right) .
$$

If $m=0$, it thus follows that $n=1$ which, with positive sign, leads us to the solution $(x, y)=(t, 1)$. If $m<0$ then necessarily $n<0$ and so

$$
\left|x-y \theta_{2}\right|=\left|\left(t-\theta_{2}\right)^{n} \theta_{2}^{-m}\right|>1,
$$

contradicting (1.4). We may thus conclude that $m>0$ (whereby also $n>0$ ). Since

and

$$
\begin{aligned}
& \log \left(t^{3}-3-\frac{\kappa_{8}}{t^{3}}\right)=3 \log t-\frac{3}{t^{3}}-\frac{\kappa_{11}}{t^{6}} \text { for } \kappa_{11} \in(4.5,7.7), \\
& \log \left(t^{3}-3-\frac{\kappa_{9}}{t^{3}}\right)=3 \log t-\frac{3}{t^{3}}-\frac{\kappa_{12}}{t^{6}} \text { for } \kappa_{12} \in(4.5,5.7)
\end{aligned}
$$

$$
\log \left(t^{9}-4 t^{6}+\kappa_{10} t^{3}\right)=9 \log t-\frac{4}{t^{3}}-\frac{\kappa_{13}}{t^{6}} \text { for } \kappa_{13} \in(2.9,3.1),
$$

we may conclude that

$$
k:=n-3 m-1=\frac{1}{3 \log t}\left(\frac{3 n-4 m-3}{t^{3}}+\frac{\kappa_{12} n-\kappa_{13} m-\kappa_{11}}{t^{6}}\right) .
$$

Since equation (3.6) readily implies that $n>2 m$, (3.7) and the fact that $n$ and $m$ are of opposite parity allows us to conclude that $k$ is an even positive integer (so that, in particular, $n \geq 3 m+3$ ). Arguing crudely, (3.7) thus implies that

$$
2 \leq \frac{1}{3 \log t}\left(\frac{5 n}{3 t^{3}}+\frac{5 n}{t^{6}}\right)
$$

and hence

$$
n=\max \{|m|,|n|\}>3.5 t^{3} \log t .
$$

3.3. Solutions of type III. Suppose now that we have a solution $(x, y)$ with $x / y$ in $I_{3}$, i.e.

$$
t^{4}-2 t-\frac{1.13}{t^{8}}<\frac{x}{y}<t^{4}-2 t-\frac{1-\frac{1}{|y|^{3}}}{t^{8}}
$$

As with solutions of type II, we may suppose that both $x$ and $y$ are positive integers (with, say, $y \geq 2$ ). We have

$$
0<\frac{x-y \theta_{1}}{x-y \theta_{2}}=\left(\frac{t-\theta_{1}}{t-\theta_{2}}\right)^{n}\left(\frac{\theta_{1}}{\theta_{2}}\right)^{-m},
$$

and hence $n$ and $m$ have the same parity. Arguing as for type II solutions, we may write

$$
\log \left(\frac{x-y \theta_{1}}{x-y \theta_{2}}\right)=\frac{1}{t^{3}}+\frac{5}{2 t^{6}}+\frac{25}{3 t^{9}}+\frac{\kappa_{14}}{t^{12}}, \text { with } \kappa_{14} \in(25.9,26.6)
$$




$$
\begin{gathered}
\log \left(\frac{t-\theta_{1}}{\theta_{2}-t}\right)=6 \log t-\frac{\kappa_{15}}{t^{3}}, \text { with } \kappa_{15} \in(2.9,3.1), \\
\log \left(\frac{\theta_{2}}{\left|\theta_{1}\right|}\right)=6 \log t-\frac{2}{t^{3}}-\frac{\kappa_{16}}{t^{6}}, \text { for } \kappa_{16} \in(-0.1,0.1),
\end{gathered}
$$

and consider the identity

$$
\log \left(\frac{x-y \theta_{1}}{x-y \theta_{2}}\right)=n \log \left(\frac{t-\theta_{1}}{\theta_{2}-t}\right)+m \log \left(\frac{\theta_{2}}{\left|\theta_{1}\right|}\right) .
$$

We thus have

$$
n+m=\frac{1}{6 \log t}\left(\frac{2 m+\kappa_{15} n+1}{t^{3}}+\frac{5 / 2+\kappa_{16} m}{t^{6}}+\frac{25}{3 t^{9}}+\frac{\kappa_{14}}{t^{12}}\right) .
$$

It is easy to show from this that $n m \neq 0$ and, indeed, that $n$ and $m$ have opposite signs. From (2.3), since necessarily $\left|x-\theta_{2} y\right|<1$, we have $m>0$ and $n<0$, say $n=-n_{0}$.

If $m \geq n_{0}+2$, then (3.9) and the inequality $t \geq 10$ imply that

$$
m-n_{0}<\frac{1}{6 \log t}\left(\frac{2 m-2.9 n_{0}+1}{t^{3}}+\frac{2.6+0.1 m}{t^{6}}\right)<\frac{m-n_{0}}{2 t^{3} \log t},
$$

a contradiction. Since $m$ and $n$ have the same parity, we may thus conclude that either $m=n_{0}$ or $n_{0} \geq m+2$. In the first case, we have

$$
\frac{\left(\kappa_{15}-2\right) m-1}{t^{3}}-\frac{5 / 2+\kappa_{16} m}{t^{6}}-\frac{25}{3 t^{9}}-\frac{\kappa_{14}}{t^{12}}=0
$$

and hence, after a little work, that $m=1, n=-1$, corresponding to the solution $(x, y)=\left(t^{4}-2 t, 1\right)$. We may therefore assume that $n_{0} \geq m+2$ and hence, from (3.9),

and so

$$
2 \leq n_{0}-m<\frac{3.1 n_{0}-1.9 m}{6 t^{3} \log t}=\frac{3.1\left(n_{0}-m\right)}{6 t^{3} \log t}+\frac{m}{5 t^{3} \log t}
$$

$$
|n|>m>4.9\left(n_{0}-m\right) t^{3} \log t \geq 9.8 t^{3} \log t .
$$

We may thus conclude, in all cases (i.e. for solutions of type I, II or III), that

$$
\max \{|m|,|n|\} \geq 3.5 t^{3} \log t .
$$

\section{LiNEAR FORMS IN LOGARITHMS}

With a lower bound upon $\max \{|m|,|n|\}$ in hand, we now turn our attention to extracting bounds for expressions of the shape (2.4). Our starting point is Siegel's identity :

$$
\left(\theta_{2}-\theta_{3}\right)\left(x-y \theta_{1}\right)+\left(\theta_{3}-\theta_{1}\right)\left(x-y \theta_{2}\right)+\left(\theta_{1}-\theta_{2}\right)\left(x-y \theta_{3}\right)=0 .
$$

From this, we have, for example, that $\frac{x-y \theta_{3}}{x-y \theta_{2}}$ not only satisfies equation (2.3), but also

$$
\frac{x-y \theta_{3}}{x-y \theta_{2}}=\frac{\theta_{1}-\theta_{3}}{\theta_{1}-\theta_{2}}+\frac{\theta_{3}-\theta_{2}}{\theta_{1}-\theta_{2}}\left(x-y \theta_{1}\right) .
$$

It follows, if $(x, y)$ is a type I solution, say, that $\frac{x-y \theta_{3}}{x-y \theta_{2}}$ and $\frac{\theta_{1}-\theta_{3}}{\theta_{1}-\theta_{2}}$ are extremely close together, whereby, from (2.3), the linear form

$$
\Lambda_{1}=\log \left(\frac{\theta_{1}-\theta_{3}}{\theta_{1}-\theta_{2}}\right)+n \log \left(\frac{t-\theta_{2}}{t-\theta_{3}}\right)-m \log \left(\frac{\theta_{2}}{\theta_{3}}\right)
$$


is necessarily small. Explicitly, we may write $\Lambda_{1}=\log \left(1+\tau_{1}\right)$, where

$$
\tau_{1}=\frac{\left(\theta_{2}-\theta_{3}\right)\left(x-y \theta_{1}\right)}{\left(\theta_{1}-\theta_{2}\right)\left(x-y \theta_{3}\right)}
$$

We have, since $m$ and $n$ are negative in (2.2) for solutions of type I, with $|m|>3|n|$,

$$
\left|\tau_{1}\right|=\frac{\theta_{3}-\theta_{2}}{\theta_{2}-\theta_{1}}\left(\frac{t-\theta_{1}}{\theta_{3}-t}\right)^{n}\left(\frac{\theta_{3}}{\left|\theta_{1}\right|}\right)^{m}<\left(t^{3}-3\right)\left(t^{3}-3\right)^{-n}\left(t^{9}-4 t^{6}\right)^{m}<t^{7.7 m},
$$

where, in the last inequality, we are assuming that $(m, n) \neq(-4,-1)$ (a case we treated earlier). From the fact that $|\log (1+z)|<2|z|$, valid for $|z|<1 / 2$, we may thus conclude that

$$
\log \left|\Lambda_{1}\right|<\log 2-7.7|m| \log t
$$

We argue similarly in the case of solutions of types II and III, considering the linear forms

$$
\Lambda_{2}=\log \left(\frac{\theta_{3}-\theta_{2}}{\theta_{2}-\theta_{1}}\right)+n \log \left|\frac{t-\theta_{1}}{t-\theta_{3}}\right|+m \log \left|\frac{\theta_{3}}{\theta_{1}}\right|
$$

and

$$
\Lambda_{3}=\log \left(\frac{\theta_{3}-\theta_{2}}{\theta_{3}-\theta_{1}}\right)+n \log \left|\frac{t-\theta_{1}}{t-\theta_{2}}\right|+m \log \left|\frac{\theta_{2}}{\theta_{1}}\right| .
$$

Corresponding to (4.2), we have, after some work, the inequalities

$$
\log \left|\Lambda_{2}\right|<\log 2-7.9 n \log t
$$

and

$$
\log \left|\Lambda_{3}\right|<\log 2-8.9|n| \log t
$$

To find lower bounds for our $\left|\Lambda_{i}\right|$, we require estimates for linear forms in logarithms of algebraic numbers. The following is the main result (Theorem 2.1) of Matveev 24:

Proposition 4.1. Let $\mathbb{K}$ be an algebraic number field of degree $D$ over $\mathbb{Q}$ and set $\chi=1$ if $\mathbb{K}$ is real and $\chi=2$ otherwise. Suppose that $\alpha_{1}, \alpha_{2}, \ldots, \alpha_{n} \in \mathbb{K}^{*}$ with absolute logarithmic heights $h\left(\alpha_{i}\right)$ for $1 \leq i \leq n$, and that

$$
A_{i} \geq \max \left\{D h\left(\alpha_{i}\right),\left|\log \alpha_{i}\right|\right\}, 1 \leq i \leq n,
$$

for some fixed choice of the logarithm. Define

$$
\Lambda=b_{1} \log \alpha_{1}+\cdots+b_{n} \log \alpha_{n},
$$

where the $b_{i}$ are rational integers and set

$$
B=\max \left\{1, \max \left\{\left|b_{i}\right| A_{i} / A_{n}: 1 \leq i \leq n\right\}\right\} .
$$

Define, further, $\Omega=A_{1} \cdots A_{n}$,

$$
\begin{gathered}
C=\frac{16}{n ! \chi} e^{n}(2 n+1+2 \chi)(n+2)(4 n+4)^{n+1}(e n / 2)^{\chi}, \\
C_{0}=\log \left(\exp (4.4 n+7) n^{5.5} D^{2} \log (e D)\right)
\end{gathered}
$$

and $W_{0}=\log (1.5 e B D \log (e D))$. Then, if $\log \alpha_{1}, \ldots, \log \alpha_{n}$ are linearly independent over $\mathbb{Z}$ and $b_{n} \neq 0$, we have

$$
\log |\Lambda|>-C C_{0} W_{0} D^{2} \Omega .
$$


We can apply this result, with suitable parameter choices, to find lower bounds upon $\Lambda_{i}$, for each $i \in\{1,2,3\}$. We will focus our attention on the case of $\Lambda_{2}$ (where the resulting upper bound upon $t$ is largest). The other cases proceed similarly; details are available from the authors on request.

To treat $\Lambda_{2}$, we choose

$$
\alpha_{3}=\frac{\theta_{3}-\theta_{2}}{\theta_{2}-\theta_{1}}, \alpha_{2}=\left|\frac{t-\theta_{1}}{t-\theta_{3}}\right|, \alpha_{1}=\left|\frac{\theta_{3}}{\theta_{1}}\right|, b_{3}=1, b_{2}=n, b_{1}=m \text { and } D=6 .
$$

From (2.1), we have

$$
\begin{aligned}
h\left(\frac{\theta_{3}-\theta_{2}}{\theta_{3}-\theta_{1}}\right) \leq 2 h\left(\theta_{3}-\theta_{2}\right) & =\frac{2}{3} \log \left(\left(\theta_{3}-\theta_{2}\right)\left(\theta_{3}-\theta_{1}\right)\left(\theta_{2}-\theta_{1}\right)\right)<6 \log t, \\
h\left(\frac{\theta_{3}}{\theta_{1}}\right) & =\frac{1}{6} \log \left(\frac{\theta_{3}}{\theta_{1}}\right)^{2}<3 \log t
\end{aligned}
$$

and

$$
h\left(\frac{t-\theta_{1}}{t-\theta_{3}}\right)=\frac{1}{6} \log \left(\frac{t-\theta_{3}}{t-\theta_{2}}\right)^{2}<3 \log t .
$$

Therefore we can take

$$
A_{3}=36 \log t, A_{1}=A_{2}=18 \log t \text { and } B=\frac{n}{2}
$$

in Proposition 4.1 to conclude that

$$
\log \left|\Lambda_{2}\right|>-8.4 \cdot 10^{15} \log ^{3} t \log (35 n) .
$$

Combining this with (4.5), we thus have that

$$
\frac{n}{\log (35 n)}<1.07 \cdot 10^{15} \log ^{2} t
$$

Appealing to (3.8), we may therefore conclude that $t \leq 576241$ (so that $n<8.9$. $\left.10^{18}\right)$.

4.0.1. Small values of $t$. At this point, there are a number of ways to proceed to handle the remaining values of $t$. One of which, which would be particularly valuable if our bound upon $t$ was less good, would be to observe that we can rewrite our linear form $\Lambda_{2}$ as

$$
\Lambda_{2}=m \log \alpha_{2}-\log \alpha_{1},
$$

where

$$
\alpha_{1}=\left(\frac{\theta_{2}-\theta_{1}}{\theta_{3}-\theta_{2}}\right)\left|\frac{t-\theta_{3}}{t-\theta_{1}}\right|^{k+1} \text { and } \alpha_{2}=\left|\frac{t-\theta_{1}}{t-\theta_{3}}\right|^{3}\left|\frac{\theta_{3}}{\theta_{1}}\right| .
$$

Since (3.7) ensures that $k$ is small, the height of $\alpha_{1}$ is not too large and so we can profitably apply lower bounds for linear forms in two logarithms, rather than three; typically, this leads to much improved numerical results.

In our case, since the values of $t$ under consideration are not especially large, we will instead appeal to a result from Diophantine approximation. Specifically, we will use a lemma of Mignotte [25, a variant of a classical result by Baker and Davenport [5]. 
Lemma 4.2. Let $\Lambda=\mu \alpha+\nu \beta+\delta$ where $\alpha, \beta$ and $\delta$ are nonzero real numbers and where $\mu, \nu$ are rational integers with $|\mu|<A$. Let $Q>0$ be a real number and suppose that $\gamma_{1}$ and $\gamma_{2}$ satisfy

$$
\left|\gamma_{1}-\frac{\alpha}{\beta}\right|<\frac{1}{100 Q^{2}} \quad \text { and } \quad\left|\gamma_{2}-\frac{\delta}{\beta}\right|<\frac{1}{Q^{2}} .
$$

Further, let $p / q$ be a rational number with $1 \leq q \leq Q$ and $\left|\gamma_{1}-p / q\right|<q^{-2}$, and suppose $q\left\|q \gamma_{2}\right\| \geq 1.01 A+2$, where $\|\cdot\|$ denotes the distance to nearest integer. Then

$$
|\Lambda|>\frac{|\beta|}{Q^{2}}
$$

To apply this lemma in our situation, we choose

$$
\alpha=\log \left|\frac{\theta_{3}}{\theta_{1}}\right|, \beta=\log \left|\frac{t-\theta_{1}}{t-\theta_{3}}\right|, \delta=\log \left(\frac{\theta_{3}-\theta_{2}}{\theta_{2}-\theta_{1}}\right), \mu=m, \nu=n
$$

and $A=3 \cdot 10^{18}$. For each $t$ with $10 \leq t \leq 576241$, we compute $\alpha, \beta$ and $\delta$ with suitable precision, choosing $Q=10^{60}$. In each case, searching the continued fraction expansion of $\gamma_{1}$, we are able to find a convergent $p / q$ with the desired properties. We may thus conclude that $\left|\Lambda_{2}\right|>10^{-120}$. On the other hand, combining (3.8) and (4.5), we have

$$
\log \left|\Lambda_{2}\right|<-27.65 t^{3} \log ^{2} t
$$

an immediate contradiction. Full details of this computation are available from the authors upon request.

\section{Closing Remarks}

It is a relatively routine exercise nowadays to solve a given cubic Thue equation (or even a fairly large number of them). Algorithmic routines, based on a paper of Tzanakis and de Weger [40, building on the foundational work of Baker 4, exist in a number of computer algebra packages, including PARI and Magma. We used code based on the latter to solve the equations corresponding to (1.4) with $|t|<10$ (where our previous arguments may fail), as well as all cubic Thue equations of the shape $F(x, y)=1$ for forms of positive discriminant $D_{F} \leq 10^{7}$. For the latter, we argued as in [10, appealing to work of Belabas [7] and Belabas and Cohen [8, 9].

For such forms, there are precisely 9 equivalence classes with $N_{F} \geq 5$ which are inequivalent to $F_{i, t}(x, y)$ for any $i \in\{1,2,3\}$ and $t \in \mathbb{Z}$. These are

\begin{tabular}{|ccl|}
\hline$F(x, y)$ & $D_{F}$ & $N_{F}$ \\
\hline$x^{3}-3 x y^{2}+y^{3}$ & 81 & 6 \\
$x^{3}+x^{2}-3 x-1$ & 148 & 5 \\
$x^{3}+2 x^{2} y-5 x y^{2}+y^{3}$ & 361 & 6 \\
$x^{3}-5 x-1$ & 473 & 5 \\
$x^{3}-7 x-1$ & 1345 & 5 \\
$x^{3}+9 x^{2}-12 x-21$ & 108729 & 5 \\
$x^{3}+21 x^{2}-2 x-21$ & 783689 & 5 \\
$x^{3}+21 x^{2}-x-22$ & 810661 & 5 \\
$x^{3}+18 x^{2}-21 x-37$ & 1257849 & 5 \\
\hline
\end{tabular}

It is also worth noting that the families of forms $F_{1, t_{1}}, F_{2, t_{2}}$ and $F_{3, t_{3}}$ are essentially disjoint. It is a routine exercise (since the discriminants in each family are 
essentially squares, whereby we may apply Runge's method) to show that the only cases where we have

$$
F_{i, t_{i}}(x, y) \sim F_{j, t_{j}}(x, y) \text { with } i, j \in\{1,2,3\}, i \neq j
$$

correspond to

$$
F_{1,0}(x, y) \sim F_{1,2}(x, y) \sim F_{2,1}(x, y) \sim F_{3,1}(x, y), \quad F_{1,4}(x, y) \sim F_{3,-1}(x, y)
$$

and

$$
F_{2,0}(x, y) \sim F_{3,0}(x, y) .
$$

Here, since $F_{1,-t}(x, y)=F_{1, t-1}(y, x)$ and $F_{2, t}(x, y)=F_{2,-t}(x, y)$ we suppose that $t_{1}, t_{2} \geq 0$.

The solution of the remaining cases of the equation $F_{2, t}(x, y)=1$, i.e those with $0 \leq t<1.35 \cdot 10^{14}$, is within computational range nowadays, requiring slight refinements of the arguments of [41], in conjunction with appeal to state-of-the-art lower bounds for linear forms in three complex logarithms, due to Mignotte [28]. The reason why this family is somewhat harder to solve is that the lower bounds upon the growth of the exponents of the corresponding fundamental units is less strong in this case. Indeed one has only that the larger exponent is of size $\gg t^{3 / 2}$. To obtain this, Wakabayashi [41] employs local arguments reminiscent of Skolem's $p$-adic method.

\section{REFERENCES}

[1] S. Akhtari. Cubic Thue Equations. Publ. Math. Debrecen. 75 (2009), 459-483.

[2] E.T. Avanesov. The representation of numbers by binary cubic forms of positive discriminant (Russian). Acta Arith. 14 (1967/68), 13-25.

[3] E.T. Avanesov. A bound for the number of representations by a special class of binary cubic forms of positive discriminant (Russian). Acta Arith. 20 (1972), 17-31.

[4] A. Baker. Contributions to the theory of Diophantine equations. I. On the representation of integers by binary forms. Philos. Trans. Roy. Soc. London Ser. A 263 (1967/1968), 173-191.

[5] A. Baker and H. Davenport. The equations $3 x^{2}-2=y^{2}$ and $8 x^{2}-7=z^{2}$. Quart. J. Math. Oxford 20 (1969), 129-137.

[6] V.I. Baulin. On an indeterminate equation of the third degree with least positive discriminant (Russian). Tul'sk Gos. Ped. Inst. Ucen. Zap. Fiz. Math. Nauk. Vip. 7 (1960), 138-170.

[7] K. Belabas. A fast algorithm to compute cubic fields. Math. Comp. 66 (1997), 1213-1237.

[8] K. Belabas and H. Cohen. Binary cubic forms and cubic number fields. Proceedings Organic Mathematics Workshop, Vancouver 1995 (CMS Conference Proceedings 20, 1997), 175-204.

[9] K. Belabas and H. Cohen. Binary cubic forms and cubic number fields. Proceedings of a Conference in Honor of A.O.L. Atkin, 1995 (AMS/IP Studies in Advanced Mathematics 7, 1998), 191-219.

[10] M.A. Bennett. On the representation of unity by binary cubic forms. Trans. Amer. Math. Soc. 353 (2001), 1507-1534.

[11] E. Bombieri and W. Schmidt. On Thue's equation. Invent. Math. 88 (1987), 69-82.

[12] A. Bremner. Integral generators in a certain quartic field and related Diophantine equations. Michigan Math. J. 32 (1985), 295-319.

[13] B.N. Delone. Über die Darstellung der Zahlen durch die binäre kubischen Formen von negativer Diskriminante. Math. Z. 31 (1930), 1-26.

[14] L.E. Dickson. History of the Theory of Numbers. Chelsea, New York, 1971.

[15] J.H. Evertse. Upper Bounds for the Numbers of Solutions of Diophantine Equations. Thesis, Amsterdam, 1983.

[16] I. Gaál and N. Schulte. Computing all power integral bases of cubic fields. Math. Comp. $\mathbf{5 3}$ (1989), 689-696.

[17] C. Heuberger. On families of parametrized Thue equations. J. Number Theory 76 (1999), $45-61$. 
[18] C. Heuberger. On general families of parametrized Thue equations. Algebraic Number Theory and Diophantine Analysis. Proceedings of the International Conference held in Graz, Austria, 1998 (F. Halter-Koch and R. F. Tichy, eds.), Walter de Gruyter, 2000, pp. 215-238.

[19] C. Heuberger, A. Togbé and V. Ziegler. Automatic solution of families of Thue equations and an example of degree 8. J. Symbolic Computation 38 (2004), 145-163.

[20] E. Lee. Studies on Diophantine Equations. PhD Thesis, Cambridge University, 1992.

[21] F. Lippok. On the representation of 1 by binary cubic forms of positive discriminant. $J$. Symbolic Computation 15 (1993), 297-313.

[22] W. Ljunggren. Einige Bemerkungen über die Darstellung ganzer Zahlen durch binäre kubische Formen mit positiver Diskriminante. Acta Math. 75 (1942), 1-21.

[23] K. Mahler. On Thue's theorem. Math. Scand. 55 (1984), 188-200.

[24] E. Matveev, An explicit lower bound for a homogeneous rational linear form in logarithms of algebraic numbers. II, Izv. Math. 64 (2000), 1217-1269.

[25] M. Mignotte. Complete resolution of some families of Diophantine equations. Number theory, Eger 1996 (De Gruyter 1998), 383-399.

[26] M. Mignotte. Verification of a conjecture of E. Thomas. J. Number Theory 44 (1993), 172177.

[27] M. Mignotte. Pethö's cubics. Publ. Math. Debrecen 56 (2000), 481-505.

[28] M. Mignotte, Linear forms in two and three logarithms and interpolation determinants. (English summary) Diophantine equations, 151-166, Tata Inst. Fund. Res. Stud. Math., 20, Tata Inst. Fund. Res., Mumbai, 2008.

[29] M. Mignotte and N. Tzanakis. On a family of cubics. J. Number Theory 39 (1991), 41-49.

[30] T. Nagell. Darstellung ganzer Zahlen durch binäre kubische Formen mit negativer Diskriminante. Math. Zeitschr. 28 (1928), 10-29.

[31] T. Nagell. Remarques sur une classe d'équations indéterminées. Arkiv för Math. 8 (1969), 199-214.

[32] R. Okazaki. Geometry of a cubic Thue Equation. Publ. Math. Debrecen. 61 (2002), 267-314.

[33] A. Pethő. On the representation of 1 by binary cubic forms with positive discriminant. Number Theory, Ulm 1987 (Springer LMN 1380), 185-196.

[34] A. Pethő. Computational methods for the resolution of Diophantine equations. Number Theory, Banff 1988 (De Gruyter 1990), 477-492.

[35] A. Pethő and R. Schulenberg. Effektives Lösen von Thue Gleichungen. Publ. Math. Debrecen (1987), 189-196.

[36] E. Thomas. Complete solutions to a family of cubic Diophantine equations. J. Number Theory 34 (1990), 235-250.

[37] E. Thomas. Solutions to certain families of Thue equations. J. Number Theory 43 (1993), 319-369.

[38] A. Thue. Über Annäherungenswerte algebraischen Zahlen. J. reine angew. Math. 135 (1909), $284-305$.

[39] N. Tzanakis. The diophantine equation $x^{3}-3 x y^{2}-y^{3}=1$ and related equations. J. Number Theory 18 (1984), 192-205.

[40] N. Tzanakis and B.M.M. de Weger. On the practical solution of the Thue equation. J. Number Theory 31 (1989), 99-132.

[41] I. Wakabayashi. On a family of cubic Thue equations with 5 solutions. Acta Arith. 109 (2003), $285-298$.

[42] V. Ziegler. Thomas' conjecture over function fields. J. Th. Nombres Bordeaux 19 (2007), 289-309.

Department of Mathematics, University of British Columbia

E-mail address: bennett@math.ubc.ca

E-mail address: amir@math.ubc.ca 